\title{
Stroke volume variation to guide fluid therapy: is it suitable for high-risk surgical patients? A terminated randomized controlled trial
}

\author{
Ib Jammer ${ }^{1,2^{*}}$, Mari Tuovila ${ }^{3}$ and Atle Ulvik ${ }^{2}$
}

\begin{abstract}
Background: Perioperative goal-directed fluid therapy (GDFT) may improve outcome after high-risk surgery. Minimal invasive measurement of stroke volume variation (SW) has been recommended to guide fluid therapy. We intended to study how perioperative GDFT with arterial-based continuous SW monitoring influences postoperative complications in a high-risk surgical population.

Methods: From February 1st 2012, all ASA 3 and 4 patients undergoing abdominal surgery in two university hospitals were assessed for randomization into a control group or GDFT group. An arterial-line cardiac output monitor was used to measure SW, and fluid was given after an algorithm in the intervention group. Restrictions of the method excluded patients undergoing laparoscopic surgery, patients with atrial fibrillation and patients with severe mitral/aortal stenosis. To detect a decrease in number of complication from $40 \%$ in the control group to $20 \%$ in the GDFT group, $n=164$ patients were needed (power $80 \%$, alpha 0.05, two-sided test). To include the needed amount of patients, the study was estimated to last for 2 years.
\end{abstract}

Results: After 1 year, 30 patients were included and the study was halted due to slow inclusion rate. Of 732 high-risk patients scheduled for abdominal surgery, 391 were screened for randomization. Of those, $n=249$ (64\%) were excluded because a laparoscopic technique was preferred and $n=95$ (24\%) due to atrial fibrillation.

Conclusions: Our study was stopped due to a slow inclusion rate. Methodological restrictions of the arterial-line cardiac output monitor excluded the majority of patients. This leaves the question if this method is appropriate to guide fluid therapy in high-risk surgical patients.

Trial registration: ClinicalTrials.gov: NCT01473446.

\section{Background}

Maintaining adequate oxygen supply to body organs is one of the main goals during anaesthesia, and giving intravenous fluid is one way to achieve this goal. A Cochrane systematic review found that complication rate and length of hospital stay, but not mortality, were reduced when global blood flow is optimized perioperatively by means of fluid and or drugs [1].

\footnotetext{
* Correspondence: ib.jammer@helse-bergen.no

'Department of Clinical Medicine, University of Bergen, 5020 Bergen, Norway ${ }^{2}$ Department of Anaesthesia and Intensive Care, Haukeland University Hospital, 5021 Bergen, Norway

Full list of author information is available at the end of the article
}

Recent studies show the development and use of several minimal invasive methods to estimate cardiac output and guide fluid therapy [2]. Despite the unclear evidence in the literature and contradictory findings in clinical trials, the pressure on clinicians to use a goaldirected fluid therapy approach is high. In the UK, there is even a governmental financial incentive for hospitals to use Oesophageal Doppler for its patients [3] because the goal-directed approach may be cost effective [4].

High-risk patient may have the greatest benefit of a goal-directed fluid approach $[5,6]$. Less capability to compensate hypo- and hypervolemia may increase the

\section{Biomed Central}

(C) 2015 Jammer et al. This is an Open Access article distributed under the terms of the Creative Commons Attribution License (http://creativecommons.org/licenses/by/4.0), which permits unrestricted use, distribution, and reproduction in any medium, provided the original work is properly credited. The Creative Commons Public Domain Dedication waiver (http:// creativecommons.org/publicdomain/zero/1.0/) applies to the data made available in this article, unless otherwise stated. 
rate of complications in a poorly optimized fluid balance $[7,8]$. Benes et al. evaluate the effect of minimal invasive cardiac output-monitored fluid therapy exclusively in a high-risk abdominal surgery population [9]. Pearse describes the use of an arterial-line cardiac output monitoring in a high-risk surgery population [10]. Both studies where done on high-risk patients, using a strict definition of "high-risk". However, we could not find a consensus in the literature about the definition of "high-risk surgery". To simplify our approach to high-risk surgery, we defined therefore ASA 3 and 4 patients as high-risk patients. Then we intended to conduct a multicentre international prospective clinical trial to study what impact goal-directed fluid therapy based on continuous SVV (stroke volume variation) monitoring has on postoperative complications in this patient group.

\section{Methods}

\section{Trial design}

We planned a two-centre, assessor concealed, prospective randomized clinical trial conducted in Norway and Finland. The trial was approved by the institutional board in Norway (2011/947/REK Vest) and Finland (EETTMK:10/2012).

\section{Participants}

From 1 February 2012 to 31 January 2013, all high-risk patients defined by ASA score 3 and 4, older than 18 years scheduled for major abdominal surgery in two university hospitals were assessed for eligibility. Patients who were able to give consent when an investigator was available were screened for eligibility. Patient undergoing liver or oesophageal surgery where not screened because they follow a more restrictive fluid regimen. Exclusion criteria after screening were the following: atrial fibrillation, severe aortic or mitral stenosis, and laparoscopic surgery or declined participation. Patients were included consecutively. Informed consent was obtained from each randomized patient.

\section{Interventions}

Patients were randomized into two groups: a control group receiving traditional fluid therapy and a group with a goal-directed fluid therapy (GDFT) regimen guided by an arterial pressure-based cardiac output device (LiDCOrapid, LiDCO Ltd, London, UK) to measure SVV [11]. For more details of the study protocol, see Additional file 1.

\section{Sample size calculation}

The complication rate for lower gastrointestinal surgery in elective patients in one of the study hospitals was $40 \%$ in a previous study [12]. In the present study, a higher complication rate was expected due to inclusion of a population with a higher morbidity. To detect a decrease in number of complication from $40 \%$ in the control group to $20 \%$ in the GDFT group, $n=164$ patients were needed (power $80 \%$, alpha 0.05 , two-sided test). It was estimated that with an approximate inclusion rate of 80 patients/year in each study centre, the study could be conducted within 2 years.

\section{Interim analysis}

Due to an unexpectedly low inclusion rate, an analysis of the exclusion factors was performed after 1 year. This resulted in termination of the study. A retrospective analysis of all patients undergoing abdominal surgery within the last year and their comorbidities and surgical techniques was undertaken after approval of the institutional board.

\section{Outcomes}

The primary endpoint was the proportion of patients suffering of one or more complication within 5 days postoperatively.

After termination of the study, we decided to reject the primary outcome due to heavy underpowered sample size. To analyse reasons for exclusion, we determined the amount of excluded patients. No statistical analysis was performed of the numbers collected.

\section{Results}

\section{Participant flow}

During 1 year, $n=732$ high-risk patients were scheduled for abdominal surgery.

Of these, $n=341$ were not screened for inclusion. Reasons were either no investigator present or equipment missing $(n=224)$, scheduled liver or oesophagus surgery ( $n=64)$ or the patient were unable to give informed consent $(n=53)$.

Of all scheduled patients, $n=391$ patients were screened for randomisation. Of these, 64 \% $(n=249)$ were excluded because a laparoscopic technique was preferred, and $24 \%(n=95)$ were excluded due to atrial fibrillation. The patient flow through the study can be seen in Fig. 1. Of all screened patients, only $7.7 \%(n=30)$ could be included in the study. The outcome data is presented by study group allocation in Table 1. A de-identified database containing all collected data of included patients is available online as an additional file (see Additional file 2).

\section{Reason for stopped trial}

After 1 year, the number of randomized patients we could include in the study was only $18 \%$ of the estimated number we have been expected at that time. We 


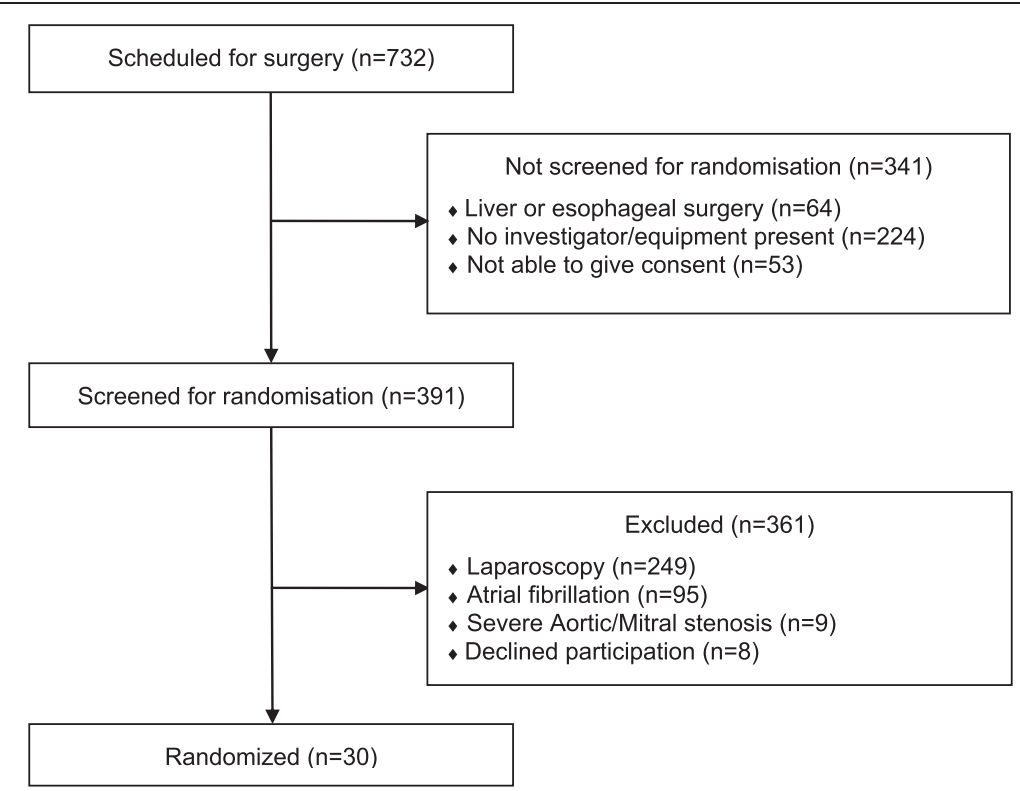

Fig. 1 Flow diagram for patients' progression through the trial

calculated that at this inclusion speed, the study would last more than 5 years and therefore decided to terminate the study early.

\section{Discussion}

\section{Principal findings}

In our study, a majority of high-risk surgical patients defined as ASA 3 and ASA 4 were not eligible for an arterial-line-based GDFT approach. The main reasons are methodological limitations of the arterial-line waveform analysis. The majority of patients had to be excluded because a laparoscopic surgical technique was preferred or due to atrial fibrillation.

This study was meant to be a prospective randomized controlled trial with a pragmatic approach to include patients. This would reflect daily routines, strengthening the study. Because the study was conducted in two tertiary hospitals, we had a high amount of high-risk surgical patients. Therefore, we expected to include enough patients in short time to run a well-powered study. Of 732 patients, 224 were not screened for randomisation due to investigator or equipment not being available. If this patient group also could have been screened, we may have had a higher number of patients randomized. However, it is to assume that the same fraction of patients would have to be excluded due to laparoscopic surgery and atrial fibrillation. Therefore, we do not believe that the total amount of patients that could be randomized would be much higher.

We terminated the study early, resulting in a heavily underpowered study. The primary outcome, postoperative morbidity, cannot be assessed since we just included
30 patients, and a statistical analysis would be meaningless. Consequently, we present just the patient flow numbers and not a complete statistical analysis of complications.

The low number of patients that could possibly benefit from GDFT is valid for our hospitals where the surgeons prefer to operate on high-risk patients with minimal invasive surgery. In hospitals that perform a higher amount of open surgery, the use of a minimal invasive GDFT approach may be more feasible.

We define the high-risk surgical patient by the ASA score to make the study pragmatic. However, other authors define "high-risk surgery" or the "high-risk patient" in different ways [13-15]. This makes comparison of trials dealing with this patient group difficult.

Maguire found in a retrospective electronic chart study of his hospital that $n=12.308$ patients underwent surgery in 1 year, but only $n=4.792$ (39\%) fulfilled the criteria for an arterial-line-based cardiac output monitor, and of these, only $23.2 \%$ had an arterial-line. There was no report on how many of the patients were ASA III/IV patients [16].

Arterial-line-based waveform analysis measures hemodynamics by calculation of stroke volume variation or pulse pressure variation. However, arterialline-based output methods are not applicable to large patient groups due to their limitations [16]. One limitation is laparoscopic procedures [17]. The increased intraabdominal pressure from the pneumoperitoneum affects dynamic parameters independently in changes of volume status [17-19]. Consequently would SVV during pneumoperitoneum increase while the blood volume do not decrease, it 
Table 1 Complications (definition) within 5 days after surgery

\begin{tabular}{|c|c|c|}
\hline & $\begin{array}{l}\text { Intervention } \\
\text { group }\end{array}$ & $\begin{array}{l}\text { Control } \\
\text { group }\end{array}$ \\
\hline & $n=14$ & $n=16$ \\
\hline \multicolumn{3}{|l|}{ Pulmonary } \\
\hline Pneumonia (x-ray + antibiotics) & 4 & 0 \\
\hline Pleural fluid (supplemental oxygen $+x$-ray) & 0 & 2 \\
\hline Atelectasis (supplemental oxygen $+x$-ray) & 3 & 1 \\
\hline Pneumothorax & 0 & 0 \\
\hline $\begin{array}{l}\text { Respiratory failure (intensive care } \\
\text { treatment) }\end{array}$ & 2 & 1 \\
\hline $\begin{array}{l}\text { Pulmonary emboli (computed tomography } \\
\text { + treatment) }\end{array}$ & 0 & 0 \\
\hline \multicolumn{3}{|l|}{ Cardial } \\
\hline $\begin{array}{l}\text { Arrytmia (electrocardiogram + treatment } \\
\text { or cardiologist consultation) }\end{array}$ & 2 & 1 \\
\hline $\begin{array}{l}\text { Coronary ischemia (electrocardiogram + } \\
\text { troponin) }\end{array}$ & 0 & 0 \\
\hline $\begin{array}{l}\text { Pulmonary stasis/oedema ( } \mathrm{x} \text {-ray or } \\
\text { treatment) }\end{array}$ & 1 & 1 \\
\hline \multicolumn{3}{|l|}{ Neurological } \\
\hline Postoperative delirium (treatment) & 1 & 2 \\
\hline Focal neurological deficit & 0 & 0 \\
\hline \multicolumn{3}{|l|}{ Infectious } \\
\hline $\begin{array}{l}\text { Wound infection (phlegmone }+ \text { antibiotics } \\
\text { or drainage) }\end{array}$ & 0 & 0 \\
\hline $\begin{array}{l}\text { Intraabdominal infection (computed } \\
\text { tomography }+ \text { antibiotics) }\end{array}$ & 0 & 0 \\
\hline Central venous catheter infection & 0 & 0 \\
\hline Wound rupture (operation) & 0 & 0 \\
\hline \multicolumn{3}{|l|}{ Gastrointestinal (Gl) } \\
\hline Mechanical ileus (operation) & 0 & 1 \\
\hline Gl bleeding (transfusion or gastroscopy) & 0 & 0 \\
\hline $\begin{array}{l}\text { Paralytic ileus (unable to tolerate enteral } \\
\text { diet }>5 \text { days) }\end{array}$ & 1 & 2 \\
\hline \multicolumn{3}{|l|}{ Others } \\
\hline $\begin{array}{l}\text { Renal impairment (creatinine increase > } \\
33 \% \text { ) }\end{array}$ & 0 & 1 \\
\hline $\begin{array}{l}\text { Impaired spontaneous voiding } \\
\text { (catheterization }>2 \text { times) }\end{array}$ & 1 & 0 \\
\hline Venous thrombosis (treatment) & 0 & 0 \\
\hline Sum of complications & 15 & 12 \\
\hline Patients with at least one complication & 7 & 7 \\
\hline
\end{tabular}

would lead to false positive readings [20]. It is therefore not well validated in humans $[21,22]$. Other limitations of waveform analysis measurements are cardiac arrhythmias and patients with severe cardiac valvulopatias [23].

Despite criticism about the evidence of the effect of goal-directed therapy, one single method of minimal invasive cardiac output monitoring (Oesophagus Doppler) has even been officially recommended in the National Institute for Health and Clinical Excellence guidelines of the UK (http://www.nice.org.uk/guidance/MTG3). This decision has been criticized due to the lack of proof $[24,25]$, and the method may not be superior to a strategy of a neutral balance [26]. Other studies have not found benefices in a goal-directed fluid approach $[10,12,26-30]$, have not found benefit using a restrictive fluid approach [31] or even have worse outcome in physically fit patients [5].

It is biologically plausible that the right amount of fluid given at the right time increase oxygen delivery to the organs and thereby benefit patient outcome. There has been a meta-analysis confirming that a GDFT approach may decrease postoperative complications. However, many included studies are small single centre studies with a high risk of bias or methodological limitations $[1,32,6]$. The effect on outcome in these studies is mostly small. An even statistical distribution of different studies with a small effect size would consequently result in a number of studies that would show no effect or even harm. The marked overweight of studies with a small positive effect on outcome may indicate a publication bias favouring trials with positive results. This may mask limitations of the arterial-line-based GDFT method that we report. Other studies investigating high-risk surgical patients do not report the exclusion rate due to atrial fibrillation when this condition restricted the GDFT method used $[5,9]$.

The OPTIMIZE trial with a study population of 734 patients is the largest trial on GDFT to date. It could not show a reduction of complications after perioperative arterial-line-based GDFT. However, when including the OPTIMIZE trial in an updated Cochrane metaanalysis, it indicates a reduced complication rate [10].

Goal-directed fluid therapy may be more important in a high-risk surgery population than in a relatively healthy population. Limitations of the method with an arterialline-based monitor may cause exclusion of a patient group who may benefit most of the treatment. In the UK, it is recommended to use an Oesophagus Doppler to guide fluid therapy preoperatively. The same limitations that apply to the arterial-line-based method (exclusion of patient with atrial fibrillation and laparoscopic procedures) would apply to this method too.

\section{Conclusions}

Our primary goal was to investigate if high-risk surgical patients benefit from SVV-guided fluid therapy. This question still remains open. A majority of our patients had to be excluded from the trial due to methodological limitations. This leaves the question whether or not an arterial-line-based cardiac output monitor is the best method to guide fluid therapy in high-risk surgical patients. 


\section{Additional files}

\section{Additional file 1: Study protocol. Study protocol, Word 2010} document.

Additional file 2: De-identified SPSS database containing collected data of all randomized patients. SPSS Statistics Data Document.

\section{Abbreviations}

ASA: American society of anesthesia score; GDFT: Goal-directed fluid therapy; SW: Stroke volume variation.

\section{Competing interests}

The authors declare that they have no competing interests.

\section{Authors' contribution}

IJ wrote the protocol and the first draft of the manuscript. IJ, MT and AU participated in the study design and data collection. All authors wrote, read and approved the final manuscript.

\section{Acknowledgements}

This study was supported by an unrestricted grant by The Eckbo Foundations, Norway and departmental funding. We thank Dr. Gro Østgaard for supporting the study and for critical appraisal of the manuscript.

\section{Author details}

${ }^{1}$ Department of Clinical Medicine, University of Bergen, 5020 Bergen, Norway. ${ }^{2}$ Department of Anaesthesia and Intensive Care, Haukeland University Hospital, 5021 Bergen, Norway. ${ }^{3}$ Department of Anesthesiology and Intensive Care, Oulu University Hospital, PL 21, 90029 Oulu, Finland.

\section{Received: 6 February 2015 Accepted: 30 June 2015}

Published online: 22 July 2015

\section{References}

1. Grocott MP, Dushianthan A, Hamilton MA, Mythen MG, Harrison D, Rowan $K$, et al. Perioperative increase in global blood flow to explicit defined goals and outcomes after surgery: a Cochrane Systematic Review. Br J Anaesth. 2013;111(4):535-48. doi:10.1093/bja/aet155.

2. Ramsingh D, Alexander B, Cannesson M. Clinical review: does it matter which hemodynamic monitoring system is used? Crit Care. 2012;17(2):208.

3. Campbell B. Innovation, NICE, and CardioQ. Br J Anaesth. 2012;108(5):726-9. doi:10.1093/bja/aes122.

4. Bartha E, Davidson T, Hommel A, Thorngren KG, Carlsson P, Kalman S. Cost-effectiveness analysis of goal-directed hemodynamic treatment of elderly hip fracture patients: before clinical research starts. Anesthesiology. 2012;117(3):519-30. doi:10.1097/ALN.0b013e3182655eb2.

5. Challand C, Struthers R, Sneyd JR, Erasmus PD, Mellor N, Hosie KB, et al. Randomized controlled trial of intraoperative goal-directed fluid therapy in aerobically fit and unfit patients having major colorectal surgery. $\mathrm{Br}$ J Anaesth. 2011;108(1):53-62. doi:10.1093/bja/aer273.

6. Cecconi M, Corredor C, Arulkumaran N, Abuella G, Ball J, Grounds RM, et al. Clinical review: goal-directed therapy-what is the evidence in surgical patients? The effect on different risk groups. Crit Care. 2013;17(2):209. doi:10.1186/cc11823.

7. Banz VM, Jakob SM, Inderbitzin D. Review article: improving outcome after major surgery: pathophysiological considerations. Anesth Analg. 2011;112(5):1147-55. doi:10.1213/ANE.0b013e3181ed114e.

8. Lees N, Hamilton M, Rhodes A. Clinical review: goal-directed therapy in high risk surgical patients. Crit Care. 2009;13:231.

9. Benes J, Chytra I, Altmann P, Hluchy M, Kasal E, Svitak R, et al. Intraoperative fluid optimization using stroke volume variation in high risk surgical patients: results of prospective randomized study. Crit Care. 2010;14(3):R118. doi:10.1186/cc9070.

10. Pearse RM, Harrison DA, MacDonald N, Gillies MA, Blunt M, Ackland G, et al. Effect of a perioperative, cardiac output-guided hemodynamic therapy algorithm on outcomes following major gastrointestinal surgery: a randomized clinical trial and systematic review. JAMA. 2014;311(21):2181-90. doi:10.1001/ jama.2014.5305.
11. Cannesson M, Aboy M, Hofer CK, Rehman M. Pulse pressure variation: where are we today? J Clin Monit Comput. 2011;25(1):45-56. doi:10.1007/ s10877-010-9229-1.

12. Jammer I, Ulvik A, Erichsen C, Lodemel O, Ostgaard G. Does central venous oxygen saturation-directed fluid therapy affect postoperative morbidity after colorectal surgery? A randomized assessor-blinded controlled trial. Anesthesiology. 2010;113(5):1072-80. doi:10.1097/ALN.0b013e3181f79337.

13. Pearse R, Harrison D, James P, Watson D, Hinds C, Rhodes A, et al. Identification and characterisation of the high-risk surgical population in the United Kingdom. Crit Care. 2006;10:R81.

14. Montenij $L$, de Waal E, Frank M, van Beest P, de Wit A, Kruitwagen C, et al. Influence of early goal-directed therapy using arterial waveform analysis on major complications after high-risk abdominal surgery: study protocol for a multicenter randomized controlled superiority trial. Trials. 2014;15:360. doi:10.1186/1745-6215-15-360.

15. Boyd O, Grounds RM, Bennett ED. A randomized clinical trial of the effect of deliberate perioperative increase of oxygen delivery on mortality in high-risk surgical patients. JAMA. 1993;270(22):2699-707.

16. Maguire S, Rinehart J, Vakharia S, Cannesson M. Technical communication: respiratory variation in pulse pressure and plethysmographic waveforms: intraoperative applicability in a North American academic center. Anesth Analg. 2011;112(1):94-6. doi:10.1213/ANE.0b013e318200366b.

17. Duperret $S$, Lhuillier F, Piriou V, Vivier E, Metton $O$, Branche $P$, et al. Increased intra-abdominal pressure affects respiratory variations in arterial pressure in normovolaemic and hypovolaemic mechanically ventilated healthy pigs. Intensive Care Med. 2007;33(1):163-71. doi:10.1007/s00134-006-0412-2.

18. Tournadre JP, Allaouchiche B, Cayrel V, Mathon L, Chassard D. Estimation of cardiac preload changes by systolic pressure variation in pigs undergoing pneumoperitoneum. Acta Anaesthesiol Scand. 2000;44(3):231-5.

19. Guenoun T, Aka EJ, Journois D, Philippe H, Chevallier JM, Safran D. Effects of laparoscopic pneumoperitoneum and changes in position on arterial pulse pressure wave-form: comparison between morbidly obese and normal-weight patients. Obes Surg. 2006;16(8):1075-81. doi:10.1381/096089206778026253.

20. Michard F, Chemla D, Teboul JL. Applicability of pulse pressure variation: how many shades of grey? Crit Care. 2015;19(1):144. doi:10.1186/s13054-015-0869-x.

21. Hoiseth LO, Hoff IE, Myre K, Landsverk SA, Kirkeboen KA. Dynamic variables of fluid responsiveness during pneumoperitoneum and laparoscopic surgery. Acta Anaesthesiol Scand. 2012;56(6):777-86. doi:10.1111/j.1399-6576.2011.02641.x.

22. Jacques D, Bendjelid K, Duperret S, Colling J, Piriou V, Viale JP. Pulse pressure variation and stroke volume variation during increased intra-abdominal pressure: an experimental study. Crit Care. 2011;15(1):R33. doi:10.1186/cc9980.

23. Chew MS, Aneman A. Haemodynamic monitoring using arterial waveform analysis. Curr Opin Crit Care. 2013;19(3):234-41. doi:10.1097/MCC.0b013e32836091ae.

24. Morris C. Oesophageal Doppler monitoring, doubt and equipoise: evidence based medicine means change. Anaesthesia. 2013;68(7):684-8. doi:10.1111/ anae.12306.

25. Ghosh S, Arthur B, Klein AA. NICE guidance on CardioQ(TM) oesophageal Doppler monitoring. Anaesthesia. 2011;66(12):1081-3. doi:10.1111/j.1365-2044.2011.06967.x.

26. Brandstrup B, Svendsen PE, Rasmussen M, Belhage B, Rodt SA, Hansen B, et al. Which goal for fluid therapy during colorectal surgery is followed by the best outcome: near-maximal stroke volume or zero fluid balance? $\mathrm{Br}$ J Anaesth. 2012:109(2):191-9. doi:10.1093/bja/aes163.

27. Srinivasa S, Taylor MH, Singh PP, YU TC, Soop M, Hill AG. Randomized clinical trial of goal-directed fluid therapy within an enhanced recovery protocol for elective colectomy. Br J Surg. 2013;100(1):66-74. doi:10.1002/bjs.8940.

28. Srinivasa S, Taylor MH, Singh PP, Lemanu DP, MacCormick AD, Hill AG. Goal-directed fluid therapy in major elective rectal surgery. Int J Surg. 2014;12(12):1467-72. doi:10.1016/j.ijsu.2014.11.010.

29. Pestana D, Espinosa E, Eden A, Najera D, Collar L, Aldecoa C, et al. Perioperative goal-directed hemodynamic optimization using noninvasive cardiac output monitoring in major abdominal surgery: a prospective, randomized, multicenter, pragmatic trial: POEMAS Study (PeriOperative goal-directed thErapy in Major Abdominal Surgery). Anesth Analg. 2014;119(3):579-87. doi:10.1213/ANE.0000000000000295.

30. Moppett IK, Rowlands M, Mannings A, Moran CG, Wiles MD, Investigators N. LiDCO-based fluid management in patients undergoing hip fracture surgery under spinal anaesthesia: a randomized trial and systematic review. Br J Anaesth. 2015;114(3):444-59. doi:10.1093/bja/aeu386. 
31. Phan TD, D'Souza B, Rattray MJ, Johnston MJ, Cowie BS. A randomised controlled trial of fluid restriction compared to oesophageal Dopplerguided goal-directed fluid therapy in elective major colorectal surgery within an Enhanced Recovery After Surgery program. Anaesth Intensive Care. 2014;42(6):752-60.

32. Hamilton MA, Cecconi M, Rhodes A. A systematic review and meta-analysis on the use of preemptive hemodynamic intervention to improve postoperative outcomes in moderate and high-risk surgical patients. Anesth Analg.

2011;112(6):1392-402. doi:10.1213/ANE.0b013e3181eeaae5.

Submit your next manuscript to BioMed Central and take full advantage of:

- Convenient online submission

- Thorough peer review

- No space constraints or color figure charges

- Immediate publication on acceptance

- Inclusion in PubMed, CAS, Scopus and Google Scholar

- Research which is freely available for redistribution 